\title{
Excisão total do mesorreto por técnica robótica: resultados cirúrgicos e oncológicos iniciais
}

\author{
Robotic total mesorectal excision: early surgical and oncological outcomes
}

\author{
TIAGO LEAL GHEZZI ${ }^{1,2}$, FABRIZIO LUCA ${ }^{2}$, MANUELA VALVO ${ }^{2}$, SABINE CENCIARELLI ${ }^{2}$, \\ SIMONETTA POZZI ${ }^{2}$, DANILO UMANA², ROBERTO BIFFI ${ }^{2}$ \\ ${ }^{1}$ Divisão de Coloproctologia, Hospital Moinhos de Vento, Porto Alegre, Brasil. \\ ${ }^{2}$ Divisão de Cirurgia Abdomino-Pélvica, Instituto Europeu de Oncologia, Milão, Itália.
}

GHEZZI TL, LUCA F, VALVO M, CENCIARELLI S, POZZI S, UMANA D, BIFFI R. Excisão total do mesorreto por técnica robótica: resultados cirúrgicos e oncológicos iniciais. Rev bras Coloproct, 2011;31(1): 26-31.

RESUMO: Objetivos: avaliar os resultados cirúrgicos e oncológicos imediatos em pacientes com câncer de reto extraperitoneal submetidos à excisão total do mesorreto (ETM) robótica. Métodos: de janeiro de 2007 a março de 2010 um total de 60 pacientes foram operados de forma consecutiva por técnica robótica. Os dados cirúrgicos e os desfechos oncológicos iniciais foram prospectivamente registrados em um banco de dados. Resultados: foram operados 35 homens e 25 mulheres. A idade média foi de 60,3 $\pm \mathbf{1 1 , 7}$ anos. Cirurgia com preservação esfincteriana foi possível em 52 pacientes. A média da amostra linfonodal foi de $18,7 \pm 8,8$ linfonodos. A média da margem cirúrgica distal foi de $2,9 \pm 1,7 \mathrm{~cm}$, enquanto a margem radial foi negativa em todos os pacientes. O tempo de seguimento pós-operatório foi de 14,3 meses. Apenas uma recidiva pélvica foi observada. As taxas de sobrevida geral e sobrevida câncer-específica foram respectivamente de 97,6\% e 98,3\%. Conclusões: ETM robótica é uma cirurgia exequível e segura. Apresenta resultados iguais ou mesmo melhores que as técnicas aberta e laparoscópica em termos de morbimortalidade, taxa de preservação esfincteriana e desfechos oncológicos iniciais.

Palavras-chave: robótica; cirurgia colorretal; procedimentos cirúrgicos minimamente invasivos; neoplasias retais.

\section{INTRODUÇÃO}

De acordo com a Conferência de Consenso Triparitário estabelecido em Washington, em 1999, a excisão total do mesorreto (ETM) consiste na ressecção completa do tecido visceral mesorretal até o nível dos músculos elevadores do ânus ${ }^{1}$. Desde a sua introdução, proposta por Heald, em 1982, as taxas de sobrevida e de recidiva pélvica em pacientes submetidos à cirurgia do câncer de reto melhoraram substancialmente ${ }^{2,3}$. Em razão disso, a ETM é considerada atualmente o tratamento cirúrgico gold standard de pacientes com tumores localizados nos terços inferior e médio do reto $^{4}$. Mais recentemente, a quimiorradioterapia neoadjuvante tem sido empregada não apenas para dimi- nuir a taxa de recidiva local, mas também para tornar possível a cirurgia de preservação esfincteriana a um maior número de pacientes ${ }^{5,6}$.

Diversos estudos demonstraram que a cirurgia laparoscópica do câncer de cólon apresenta resultados imediatos superiores e resultados oncológicos equivalentes à cirurgia aberta ${ }^{7,8,9}$. Apesar dos poucos ensaios clínicos randomizados, a cirurgia laparoscópica do câncer de reto apresenta resultados pós-operatórios e oncológicos similares à cirurgia aberta ${ }^{9-17}$. Apesar de suas vantagens, a complexidade técnica da excisão total do mesorreto, a longa curva de aprendizado e a possibilidade de que ambos os fatores possam comprometer os resultados oncológicos são os principais fatores que limitam a ampla aplicação da cirurgia

Fonte de auxílio à pesquisa: Fundação Umberto Veronesi, Instituto Europeu de Oncologia, Milão, Itália. Conflito de interesse: nada a declarar. 
laparoscópica do reto ${ }^{17,18}$. A cirurgia robótica poderá superar essas limitações, proporcionando ao cirurgião vantagens como a filtragem do tremor, a maior precisão de movimentos, a visualização tridimensional e estável do campo operatório e a reprodução dos movimentos do punho humano ${ }^{19}$.

Os objetivos deste estudo são avaliar os resultados cirúrgicos imediatos, bem como os desfechos oncológicos iniciais, em pacientes com câncer de reto extraperitoneal submetidos à ETM por técnica cirúrgica robótica.

\section{PACIENTES E MÉTODOS}

Entre janeiro de 2007 e março de 2010, um total de 60 pacientes com adenocarcinoma dos terços inferior e médio do reto foram submetidos de forma consecutiva à ressecção por técnica robótica usando o Sistema Cirúrgico da Vinci S HD. Os cânceres de reto inferior e médio foram definidos por sua localização, respectivamente, de 0 a $5 \mathrm{~cm}$ e de 5,1 a $10 \mathrm{~cm}$ da margem anal. Foram excluídos deste estudo pacientes com diagnóstico de câncer T4 durante o estadiamento pré-operatório, com tipo histológico não-adenocarcinoma ou submetido a qualquer tipo de ressecção multivisceral. Todos os pacientes receberam uma detalhada explicação do procedimento e assinaram um termo de consentimento informado. Os procedimentos foram executados por dois cirurgiões com grande experiência em cirurgia oncológica colorretal por técnica convencional. Dados epidemiológicos, clínicos, cirúrgicos e patológicos foram coletados e inseridos prospectivamente em um banco de dados. O seguimento pós-operatório foi realizado através de consultas médicas semestrais nos dois primeiros anos e anuais do terceiro ao quinto ano. Tomografia computadorizada de abdome e tórax e dosagem do antígeno carcinoembriônico (CEA) e CA 19.9 foram realizadas de formas alternadas com radiografia de tórax, ecografia de abdome, dosagem do CEA e CA 19.9 a cada consulta. Retossigmoidoscopia de controle foi executada no sexto mês de seguimento, enquanto uma colonoscopia foi realizada a cada dois anos a partir do primeiro ano.

\section{RESULTADOS}

Entre janeiro de 2007 e março de 2010, um total de 60 pacientes ( 35 homens e 25 mulheres) com câncer de reto extraperitoneal foram submetidos à excisão total do mesorreto por técnica robótica. A idade média dos pacientes foi de $60,3 \pm 11,7$ anos $(37$ a 82 anos). Os dados demográficos, clínicos e cirúrgicos dos pacientes encontram-se na Tabela 1. Trinta e um pacientes eram portadores de câncer do reto médio e 29 pacientes de câncer do reto inferior. A média da distância da borda inferior do tumor à margem anal foi de $6,0 \pm 2,5 \mathrm{~cm}$. Cirurgia com preservação esfincteriana foi possível em 52 dos 60 pacientes estudados. Em 50 pacientes foi executada uma ostomia em alça para derivação fecal temporária, sendo 46 ileostomias e quatro colostomias. Oito pacientes foram submetidos à amputação abdominoperineal de reto, 41 pacientes à ressecção anterior de reto e 11 pacientes à ressecção anterior de reto com ressecção interesfincteriana e anastomose coloanal. Os pacientes apresentaram a

Tabela 1. Dados epidemiológicos, clínicos e cirúrgicos.

\begin{tabular}{|c|c|}
\hline Características & $\mathrm{N}=60(\%)$ \\
\hline \multicolumn{2}{|l|}{ Sexo (n) } \\
\hline Masculino & $35(58,3 \%)$ \\
\hline Feminino & $25(41,7 \%)$ \\
\hline Idade $(\operatorname{anos})^{*}$ & $60,3 \pm 11,7$ \\
\hline Indice de massa corporal $\left(\mathrm{kg} / \mathrm{m}^{2}\right)^{*}$ & $24,8 \pm 4,2$ \\
\hline \multicolumn{2}{|l|}{ Escore ASA (n) } \\
\hline 1 & $10(16,7 \%)$ \\
\hline 2 & $45(75 \%)$ \\
\hline 3 & $5(8,3 \%)$ \\
\hline \multicolumn{2}{|l|}{ Cirurgia abdominal prévia (n) } \\
\hline Sim & $25(41,7 \%)$ \\
\hline Não & $35(58,3 \%)$ \\
\hline \multicolumn{2}{|l|}{ Quimiorradioterapia neoadjuvante (n) } \\
\hline Sim & $48(80 \%)$ \\
\hline Não & $12(20 \%)$ \\
\hline \multicolumn{2}{|l|}{ Localização do tumor (n) } \\
\hline Terço médio do reto $(5,1$ a $10 \mathrm{~cm})$ & $31(51,7 \%)$ \\
\hline Terço inferior do reto $(0$ a $5 \mathrm{~cm})$ & $29(48,3 \%)$ \\
\hline \multicolumn{2}{|l|}{ Procedimento cirúrgico (n) } \\
\hline RAR & $41(68,4 \%)$ \\
\hline RAR + Interesfincteriana + Coloanal & $11(18,3 \%)$ \\
\hline Amputação abdominoperineal de reto & $8(13,3 \%)$ \\
\hline
\end{tabular}


seguinte distribuição quanto ao escore ASA: 10 pacientes ASA 1, 45 pacientes ASA 2 e 5 pacientes ASA 3. O índice de massa corporal foi de $24,8 \pm 4,2 \mathrm{~kg} / \mathrm{m}^{2}$. Vinte e cinco pacientes tinham história de cirurgia abdominal prévia. Quarenta e oito pacientes receberam tratamento quimiorradioterápico neoadjuvante.

Os dados cirúrgicos encontram-se na Tabela 2. O tempo cirúrgico médio foi de $301 \pm 59,6 \mathrm{minu}-$ tos (156 a 448 minutos) e nenhum procedimento foi convertido para técnica aberta. A mediana da perda sanguínea transoperatória estimada foi de $130,3 \mathrm{ml}$, variando de 0 a $1500 \mathrm{ml}$. Nenhum paciente necessitou de transfusão sanguínea no transoperatório. A mediana do tempo de internação pós-operatória foi de 6 dias (3 a 26 dias). Os tempos para início da eliminação de flatus e para o início da dieta líquida via oral foram respectivamente de 1,9 $\pm 1,7$ dias e $2,2 \pm$ 1,7 dias. Vinte e três pacientes desenvolveram alguma complicação pós-operatória. Apenas um paciente desenvolveu deiscência anastomótica. Este paciente, juntamente com um outro complicado com hemoperitônio pós-operatório, são os únicos dois que necessitaram de reintervenção cirúrgica. Nenhum paciente necessitou de readmissão hospitalar nos primeiros 30 dias após a cirurgia. Apenas um paciente recebeu transfusão sanguínea pós-operatória. Não houve mortalidade relacionada à cirurgia.

Tabela 2. Dados cirúrgicos.

\begin{tabular}{lc}
\hline Resultados cirúrgicos & $\mathbf{N}=\mathbf{6 0}(\mathbf{\%})$ \\
\hline Tempo cirúrgico (min)* & $301 \pm 59,6$ \\
$\begin{array}{l}\text { Sangramento transoperatório estimado } \\
\text { (mL)** }\end{array}$ & 130,3 \\
$\begin{array}{l}\text { Transfusão sanguínea transoperatória } \\
\text { (n) }\end{array}$ & $0(0 \%)$ \\
Conversão (n) & $0(0 \%)$ \\
$\begin{array}{l}\text { Ostomia de derivação*** (n) } \\
\text { Tempo de internação pós-operatório } \\
\text { (dias)** }\end{array}$ & $50(83,3 \%)$ \\
$\begin{array}{l}\text { Transfusão sanguínea pós-operatória (n) } \\
\text { Reintervenção (n) }\end{array}$ & $1(1,7 \%)$ \\
$\begin{array}{l}\text { Qualquer complicação pós-operatória } \\
<30 \text { dias (n) }\end{array}$ & $23(3,3 \%)$ \\
Mortalidade $<30$ dias (n) & $0(0 \%)$ \\
\hline *Dados apresentados como média e desvio-padrão; **Dados \\
$\begin{array}{l}\text { apresentados como mediana e valor máximo e minimo; } \\
\text { ***Excluindo-se amputaçóes abdominoperineais de reto. }\end{array}$
\end{tabular}

Quanto ao estadiamento patológico do American Joint Committee on Cancer (AJCC), foram observados 12 pacientes estadio I, 23 pacientes estadio II, 20 pacientes estadio III e cinco pacientes estadio IV (Tabela 3). A média da amostra linfonodal foi de 18,7 \pm 8,8 linfonodos, enquanto a margem radial foi negativa em todos os pacientes operados. A média da medida da margem cirúrgica distal foi de $2,9 \pm 1,7 \mathrm{~cm}$.

O tempo médio de seguimento pós-operatório dos pacientes estudados foi de 14,3 meses. Apenas um paciente $(1,7 \%)$ desenvolveu recidiva pélvica, a qual foi tratada cirurgicamente através de uma evisceração pélvica posterior por técnica aberta. As taxas de sobrevidas geral e câncer-específica acumuladas neste período são de respectivamente $97,6 \%$ e $98,3 \%$ (Tabela 3). A curva de sobrevida geral é representada na Figura 1.

\section{DISCUSSÃO}

Neste estudo descrevemos a aplicação da cirurgia robótica no tratamento do câncer do reto extraperitoneal, de acordo com os princípios da ETM, em uma série consecutiva de pacientes operados por dois cirurgiões em um centro de referência em cirurgia oncológica colorretal. No contexto do manejo minimamente invasivo do câncer de reto, são considerados pontos críticos a preservação esfincteriana, a morbidade cirúrgica, a radicalidade de ressecção e as taxas de recorrência local e de sobrevida acumulada.

Tabela 3. Dados patológicos e resultados oncológicos.

\begin{tabular}{ll}
\hline Dados patológicos e oncológicos & $\mathbf{N}=\mathbf{6 0}(\mathbf{\%})$ \\
\hline Estadio patológico AJCC (n) & $12(20 \%)$ \\
I & $23(38,4 \%)$ \\
II & $20(33,3 \%)$ \\
III & $5(8,3 \%)$ \\
IV & $18,7 \pm 8,8$ \\
Número de linfonodos (n)* & $2,9 \pm 1,7$ \\
Margem distal (cm)* & $60(100 \%)$ \\
Margem radial negativa (n) & $1(1,7 \%)$ \\
Recidiva local (n) & $97,6 \%$ \\
Sobrevida geral & $98,3 \%$ \\
Sobrevida câncer-específica &
\end{tabular}

* Dados apresentados como média e desvio-padrão; AJCC: American Joint Committee on Cancer. 


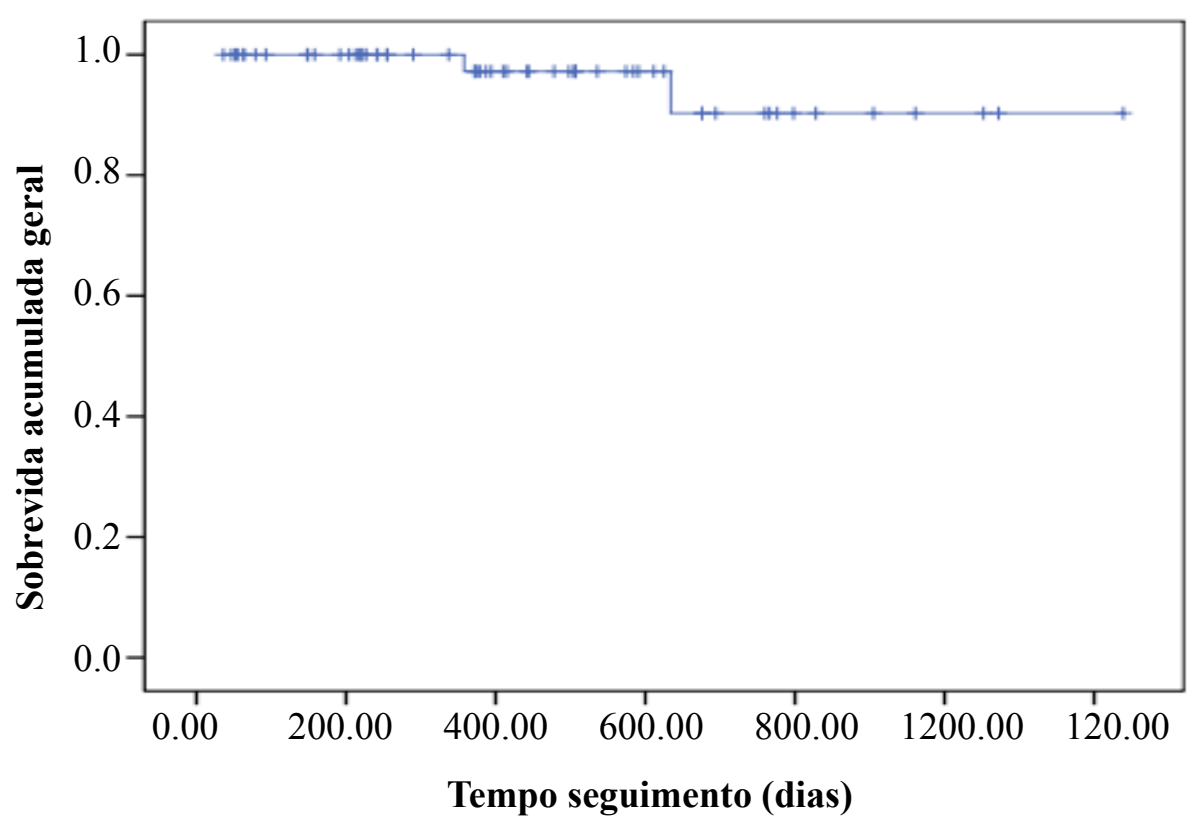

$\neg$ Survival Function

+ Censored

Figura 1 - Curva de Kaplan-Meier de sobrevida geral acumulada.

Em nosso estudo, todos os pacientes com suspeita clínica (deiscência clinica) ou com alteração do material de drenagem, mesmo na ausência de sintomatologia (deiscência subclínica), foram submetidos a enema com contraste hidrossolúvel ou retoscopia para confirmação do diagnóstico. Nos 52 pacientes submetidos à cirurgia com preservação esfincteriana, apenas um $(1,7 \%)$ desenvolveu deiscência anastomótica. Apesar dos diversos critérios adotados por diferentes autores, nossa taxa de deiscência anastomótica é inferior àquela encontrada na literatura, que varia de 3 até $17 \%$ para pacientes submetidos à ETM com preservação esfincteriana por técnica aberta, laparoscópica ou robótica ${ }^{2,20-25}$.

De acordo com a recomendação da AJCC, é necessária uma amostra linfonodal de no mínimo 10 a 14 linfonodos para um adequado estudo histopatológico. Aceita-se um número ainda menor em pacientes submetidos à quimiorradioterapia pré-operatória ${ }^{26}$. Em nossa série, apesar de $80 \%$ dos pacientes terem sidos submetidos a tratamento neoadjuvante, o número médio de linfonodos ressecados foi de 18,7. Nosso resultado, em termos de amostragem linfonodal, é superior quando comparados aqueles reportados em outras séries de ETM por técnica aberta, laparoscópica ou robótica ${ }^{2,3,20-24,27,28}$. Diversos estudos não randomizados demonstraram que a ETM com margem radial livre de invasão tumoral reduz a taxa de recidiva lo$\mathrm{cal}^{26}$. Em nosso estudo, nenhum paciente apresentou margem radial comprometida. A margem cirúrgica distal média de nossos pacientes $(2,9 \mathrm{~cm})$ atende as recomendações atuais e se assemelha aos valores observados em outras séries de cirurgia minimamente invasiva ${ }^{21,23,24,27,28}$. Ao nosso ver, esses achados refletem a radicalidade oncológica da cirurgia robótica e traduzem as vantagens oferecidas pelo robô da Vinci na dissecção de estruturas pélvicas profundas.

Uma vez respeitada a radicalidade da ressecção cirúrgica, o objetivo secundário no tratamento curativo do câncer de reto é a preservação esfincteriana. Estudo de Temple et al demonstrou que idade, grau de fixação tumoral, altura do tumor, história de radioterapia pré-operatória e experiência da equipe cirúrgica são fatores preditores de preservação esfincteriana ${ }^{29}$. Em nossa casuística, a preservação do esfíncter anal foi possível em 52 pacientes (80\%). Na nossa experiência, as cirurgias de escolha são a ressecção anterior de reto com anastomose término-terminal, segundo técnica de duplo grampeamento tipo Knight-Griffen, ou, nos pacientes com tumores muito distais, a ressecção interesfincteriana com anastomose coloanal manual.

Atualmente o tratamento gold standard do câncer de reto extraperitoneal compreende a aplicação de 
quimiorradioterapia neoadjuvante em pacientes com estadiamento pré-operatório T3-4N+ e a execução da rotineira ETM. Este tratamento é acompanhado de uma taxa de recidiva local inferior a $10 \%$ e oferece taxas de sobrevida em 5 anos de até $80 \%{ }^{4,20}$. Os desfechos oncológicos iniciais na nossa casuística, embora ainda com período de seguimento pós-operatório bastante breve (14,3 meses), podem ser considerados encorajantes. De fato, nossa taxa de recidiva local $(1,7 \%)$ é inferior àquela citada por Ridgway em sua revisão de 13 séries históricas com tempo de seguimento de 24 meses na era pós-ETM ${ }^{4}$. Conscientes da brevidade do seguimento pós-operatório até o momento, ressaltamos que as taxas de sobrevida geral e sobrevida câncer-específica mantêm-se acima de 97\%. Acreditamos que a cirurgia robótica permita a dissecção precisa dos planos anatômicos do mesorreto e, associada à quimiorradioterapia neoadjuvante, contribua para a excelência dos resultados.

\section{CONCLUSÕES}

A ETM por técnica robótica em pacientes com câncer do reto extraperitoneal é uma cirurgia exequível e segura. Apresenta resultados iguais ou até mesmo melhores que as técnicas aberta e laparoscópica no que diz respeito à morbimortalidade, radicalidade cirúrgica, preservação esfincteriana e desfechos oncológicos a curto prazo. Estudos prospectivos controlados com seguimento mais longo são necessários para avaliar se os resultados oncológicos imediatos traduzem-se a longo prazo na melhoria sustentada do controle local da doença e em melhores taxas de sobrevida.

\footnotetext{
ABSTRACT: Objectives: to evaluate the surgical and oncological outcomes of patients with extraperitoneal rectal cancer who underwent robotic total mesorectal excision (TEM). Methods: from January 2007 to March 2010 a total of 60 patients were consecutively operated on through robotic technique. Data regarding surgical data and oncological outcomes were prospectively registered in a database. Results: 35 men and 25 women underwent surgery. The mean age was $60.3 \pm 11.7$ years. Sphincter preserving surgery was possible in 52 patients. The mean number lymph node harvested was $18.7 \pm 8.8$ lymph nodes. The mean distal surgical margin was 2.9 $\pm 1.7 \mathrm{~cm}$, while the radial margin was negative in all patients. The duration of postoperative follow-up was 14.3 months. Only one local recurrence was observed. The overall and the cancer-specific survival were respectively $97.6 \%$ and $98.3 \%$. Conclusions: robotic TEM is feasible and safe. It is equal or superior to open and laparoscopic techniques in terms of morbidity and mortality rates, sphincter preservation rates and early oncological outcomes.
}

Keywords: robotics; colorectal surgery; minimally invasive surgical procedures; rectal neoplasms.

\section{REFERÊNCIAS}

1. Lowry AC, Simmang CL, Boulos P, Farmer KC, Finan PJ, Hyman N, et al. Consensus statement of definitions for anorectal physiology and rectal cancer: report of the Tripartite Consensus Conference on Definitions for anorectal physiology and rectal cancer. Washington, DC, May 1, 1999. Dis Colon Rectum 2001; 44(7):915-919.

2. Kim JS, Hur H, Kim NK, Kim YW, Cho SY, Kim JY, et al. Oncologic outcomes after surgery following preoperative chemorradiotherapy for locally advanced lower rectal cancer: abdominoperineal resection versus sphincter-preserving procedure. Ann Surg Oncol 2009;16(5):1266-73.

3. Denoya P, Wang H, Sands D, Nogueras J, Weiss E, Wexner SD. Short-term outcomes of laparoscopic total mesorectal excision following neoadjuvant chemoradiotherapy. Surg Endosc 2010;24(4):933-38.

4. Ridgway PF, Darzi AW. The role of total mesorectal excision in the management of rectal cancer. Cancer Control 2003; 10(3):205-11.
5. Wagman R, Minsky BD, Cohen AM, Guillem JG, Paty PP. Sphincter preservation in rectal cancer with preoperative radiation therapy and coloanal anastomosis: long-term follow-up. Int J Radiat Oncol Biol Phys 1998;42(1):51-7.

6. Rouanet P, Fabre JM, Dubois JB, Dravet F, Saint Aubert B, Pradel J, et al. Conservative surgery for low rectal carcinoma after high-dose radioation. Functional and oncologic results. Ann Surg 1995;221(1):67-73.

7. Veldkamp R, Gholghesaei M, Bonjer HJ, Meijer DW, Buunen $\mathrm{M}$, Jeekel J, et al. Laparoscopic resection of colon cancer. Consensus of the European Association of Endoscopic Surgery (E.A.E.S). Surg Endosc 2004;18(8):1163-85.

8. The Clinical Outcomes of Surgical Therapy Study Group. A comparision of laparoscopically assisted and open colectomy for colon cancer. N Eng J Med 2004;350(20):2050-9.

9. Guillou PJ, Quirke P, Thorpe H, Walker J, Jayne DG, Smith $\mathrm{AM}$, et al. Short-term endpoints of conventional versus laparoscopic-assisted surgery in patients with colorectal cancer (MRC CLASSIC trial): multicentre, randomised controlled trial. Lancet 2005;365(9472):1718-26. 
10. Leung KL, Kwok SP, Lam SC, Lee JF, Yiu RY, Ng SS, et al. Laparoscopic resection of rectosigmoid carcinoma: prospective randomised trial. Lancet 2004; 363(9416):1187-92.

11. Zhou ZG, Hu M, Li Y, et al. Laparoscopic versus open total mesorectal excision with anal sphincter preservation for low rectal cancer. Surg Endosc 2004;18(8):1211-15.

12. Morino M, Parini U, Giraudo G, Salval M, Contul RB, Garrone C. Laparoscopic total mesorectal excision: a consecutive series of 100 patients. Ann Surg 2003:237(3):335-42.

13. Leroy J, Jamali F, Forbes L, Smith M, Rubino F, Mutter $\mathrm{D}$, et al. Laparoscopic total mesorectal excision (TME) for rectal cancer surgery: long-term outcomes. Surg Endosc 2004;18(2):281-9.

14. Bärlehner E, Benhidjeb T, Anders S, Schike B. Laparoscopic resection for rectal cancer: outcomes in 194 patients and review of the literature. Surg Endosc 2005;19(6):757-66.

15. Hartley JE, Mehigan BJ, Qureshi AE, Duthie GS, Lee PW, Monson JR. Total mesorectal excision: assesment of the laparoscopic approach. Dis Colon Rectum 2001;44(3):315-21.

16. Morino M, Allaix ME, Giraudo G, Corno F, Garrone C. Laparoscopic versus open surgery for extraperitoneal rectal cancer: a prospective comparative study. Surg Endosc 2005;19(11):1460-7.

17. Aziz O, Constantinides V, Tekkis PP, Athanasiou T, Purkayastha S, Paraskeva P, et al. Laparoscopic versus open surgery for rectal cancer: a meta-analysis. Ann Surg Oncol 2006;13(3):413-24.

18. Kuhry E, Schwenk W, Gaupset R, Romild U, Bonjer J. Longterm outcome of laparoscopic surgery for colorectal cancer: a cochrane systematic review of randomised controlled trials. Cancer Treat Rev 2008;34(6):498-504.

19. Hance J, Rockall T, Darzi A. Robotics in colorectal surgery. Dig Surg 2004;21(5-6):339-43.

20. Veenhof AAFA, Engel AF, Craanen ME, Meijer S, de Langede Klerk ES, van der Peet DL, et al. Laparoscopic versus open total mesorectal excision: a comparative study on shortterm outcomes. A single-institution experience regarding anterior resections and abdominoperineal resections. Dig Surg 2007;24(5):367-74.
21. Baek JH, McKenzie S, Garcia-Aguilar J, Pigazzi A. Oncologic outcomes of robotic-assisted total mesorectal excision for the treatment of rectal cancer. Ann Surg 2010;251(5):882-6.

22. Choi DJ, Kim SH, Lee PJ, Kim J, Woo SU. Single-stage totally robotic dissection for rectal cancer surgery: technique and short-term outcome in 50 consecutive patients. Dis Colon Rectum 2009;52(11):1824-30.

23. Hellan M, Anderson C, Ellenhorn JD, Paz B, Pigazzi A. Short-term outcomes after robotic-assisted total mesorectal excision for rectal cancer. Ann Surg Oncol 2007; 14(11):3168-73.

24. Pigazzi A, Luca F, Patriti A, Valvo M, Ceccarelli G, Casciola L, et al. Multicentric study on robotic tumor-specific mesorectal excision for the treatment of rectal cancer. Ann Surg Oncol 2010; 17(6):1614-20.

25. Chiappa A, Biffi R, Bertani E, Zbar AP, Pace U, Crotti C, et al. Surgical outcomes after mesorectal excision for rectal cancer. J Surg Oncol 2006;94(3):182-93.

26. Colon and rectum. AJCC Cancer Staging Handbook. 2010; $7^{\text {th }}$ edition. Available from: URL: http://www.springer.com/ medicine/surgery/cancer+staging?SGWID=0-40654-0-0-0. [Cited 2010 may 27].

27. Ng KH, Lim YK, Ho KS, Ooi BS, Eu KW, et al. Roboticassisted surgery for low rectal dissection: from better views to better outcome. Singapore Med J 2009;50(8):763-7.

28. Tjandra JJ, Kilkenny JW, Buie WD, Hyman N, Simmang C, Anthony T, et al. Practice parameters for the management of rectal cancer (revised). Dis Colon Rectum 2005 Mar;48(3):411-23.

29. Temple LK, Romanus D, Niland J, Veer AT, Weiser MR, Skibber J, et al. Factors associated with sphincter-preserving for rectal cancer at National Comprehensive Cancer Network Centers. Ann Surg 2009;250(2):260-7.

\section{Endereço para correspondência:} TIAGO LEAL GHEZZI

Avenida Ramiro Barcelos, 910/601 - Bairro Floresta CEP: 90035-001 - Porto Alegre (RS), Brasil

E-mail: tlghezzi@terra.com.br; tiago.lealghezzi@ieo.it 